\title{
The Changes in Serum Insulin, Aldosterone and Endothelin-1 Levels After 3 Months of Tai Chi Chuan Training in the Adults
} Wan-An Lu ${ }^{1,2}$ and Cheng-Deng Kuo ${ }^{2 *}$

${ }^{1}$ Institute of Cultural Asset and Reinvention, Fo-Guang University, Ilan, Taiwan

${ }^{2}$ Laboratory of Biophysics, Department of Medical Research, Taipei Veterans General Hospital, Taipei, Taiwan

\begin{abstract}
Introduction: This study investigated the effects of 3 months of Yang's Tai Chi Chuan (TCC) training on the hemodynamics, fasting blood sugar (FBS), blood lipids, serum insulin, serum aldosterone, and serum endothelin-1 $(\mathrm{ET}-1)$ in the adults.

Methods: Twenty normal controls and 22 TCC trainees were recruited to participate in this study. The hemodynamics, FBS, blood lipid, serum insulin, aldosterone, ET-1, HOMA-IR, $\log (\mathrm{HOMA}), \mathrm{QUICKI}$, and TG/HDL-C before and after 3 months of TCC were compared between the 2 groups of subjects.

Results: After 3 months of TCC training, the HDL-C, QUICKI, ET-1, and aldosterone were significantly increased, whereas the serum insulin, HOMA, and $\log (\mathrm{HOMA})$ were significantly decreased in the TCC group, as compared with the control group. Intra-group comparison shows that the HDL-C, QUICKI and aldosterone were significantly increased, while the serum insulin, HOMA-IR, $\log (\mathrm{HOMA})$, and ET-1 were significantly decreased after 3 months of TCC in the TCC group. The \%FBS correlated significantly and negatively with the \%TG/HDL-C, \%QUICKI, and \%ET1. The \%Insulin correlated significantly and positively with \%HOMA, and significantly and negatively with \%QUICKI.

Conclusion: TCC training for 3 months can increase the serum levels of ET-1 and aldosterone, and decrease serum insulin level and insulin resistance in the TCC trainees. TCC might improve the cardiovascular function and insulin sensitivity of its practitioners through regulation of lipid and glucose metabolism, and the interplay among insulin, aldosterone, and ET-1.
\end{abstract}

Keywords: Tai chi chuan; Endothelin-1; Insulin; Aldosterone; HOMA-IR; QUICKI

\section{Introduction}

Low-velocity and low-impact exercises are preferred to reduce the cardiovascular and orthopedic complications in the elderly [1]. Goble et al. [2] reported that lower-intensity exercise might have similar training effects as higher-intensity exercise for cardiac patients. As an integral part of most cardiac rehabilitation programs, regular exercise can remodel the cardiovascular system and modify the autonomic nervous control of the subject [3]. For patients after acute myocardial infarction referred for cardiac rehabilitation, low- and high-intensity exercise training have been shown to improve the functional capacity and produce relatively similar changes in cardiorespiratory variables during the initial 3 months of exercise training [4]. It has been suggested that supervised exercise in the elderly should emphasize on aerobic, strengthening and flexibility training [5].

Endothelin-1 (ET-1) is a potent vasoconstrictor peptide that can be produced from the biologically inactive 38 -amino acid intermediate via cleavage by the ET-converting enzyme [6]. It was firstisolated from the culture supernatant of porcine aortic endothelial cell in 1988 [7]. Some evidences have demonstrated that ET-1 may be involved in the pathogenesis of hypertension [8] and atherosclerosis [9]. The circulating ET-1 may act as a paracrine factor in the metabolism of blood glucose through the modulation of serum insulin level [10-13]. Elevated plasma level of ET-1 has been observed in patients with atherosclerosis [9], diabetes mellitus [10], chronic heart failure [14], essential hypertension [15], chronic renal failure [16], and myocardial infarction [17]. Both the increase in plasma ET-1 and the decrease in plasma NOx may affect myocardial blood flow in patients with coronary slow flow [18].
Circulating aldosterone may directly affect vascular tone and may be part of the vasoconstriction response [19]. Tostes et al. [20] suggested that renal damage in aldosterone-dependent hypertension is associated with inflammatory processes that are mediated in part via ET-1.

Tai Chi Chuan (TCC) is a traditional oriental conditioning exercise or calisthenics that can delay the decline of aerobic power [21], is beneficial to the cardiopulmonary function [22,23], strength [23], and balance [24] of the subjects, and can reduce tension, anxiety, and mood disturbance [25]. Sun et al. [26] pointed that regular and more than 6 months' Tai Chi exercises had a beneficial effect to health-related quality of life, reducing psychological distress, promoting resilience, and reducing the BMI and blood pressure level in heart-failure patients. Xiong et al. [27] showed that the skill level of TCC may have considerable impact on metabolic and cardiorespiratory responses to TCC performance. It has been estimated that more than one hundred million people were practicing TCC regularly in the world [28].Thus, the aim of this study was to investigate the effect of 3 months of TCC training on the serum ET-1, serum aldosterone, serum insulin, fasting blood sugar, blood lipids in healthy adults.

${ }^{*}$ Corresponding author: Cheng-Deng Kuo, Laboratory of Biophysics, Departmen of Medical Research, Taipei Veterans General Hospital, Taipei, Taiwan 112, Taiwan, Tel: 886-2-28757745; Fax: 886-2-28710773; E-mail: cdkuo23@gmail.com

Received April 12, 2015; Accepted May 28, 2015; Published May 30, 2015

Citation: Lu WA, Kuo CD (2015) The Changes in Serum Insulin, Aldosterone and Endothelin-1 Levels After 3 Months of Tai Chi Chuan Training in the Adults. $J$ Diabetes Metab 6: 557. doi:10.4172/2155-6156.1000557

Copyright: () 2015 Lu WA, et al. This is an open-access article distributed unde the terms of the Creative Commons Attribution License, which permits unrestricted use, distribution, and reproduction in any medium, provided the original author and source are credited. 


\section{Material and Methods}

\section{Subject selection and study design}

Both normal subjects without TCC experience and TCC exercisers were recruited to participate in this study. The normal subjects were recruited from the community, and the TCC exercisers were recruited from a TCC training center in Taiwan. All subjects included in this study were over 50 years of age, had no previous experience with TCC, had normal lifestyles, and were capable of daily activities without limitations. Subjects who had major cardiopulmonary disease or were on regular medicine for diabetes mellitus, hypertension, renal or liver disease were not included in the study. The Institute Review Board of the hospital approved this study, and the procedure was fully explained to the subjects, and signed informed consent was obtained from them prior to the study.

Before measurements were taken, the subject took a rest in supine position for 5 minutes before the measurement of blood pressures (OMRON, HEM -770A). The hemodynamic data such as systolic blood pressure (SBP), diastolic blood pressure (DBP), mean arterial blood pressure $(\mathrm{MABP})$, and pulse pressure $(\mathrm{PP})$ were obtained from every subject in the control and TCC groups before TCC training and after 3 months of TCC training. The TCC trainee exercised TCC once per day for 3 months.

The participants of this study were requested to not take any caffeinated or alcoholic beverages for at least 24 hours prior to the study. They were also requested to not exercise on the day of study. The hemodynamic data were taken after the subject had rested quietly for 5 minutes, and then a blood sample was withdrawn for later assay. After hemodynamic measurements, the TCC trainee was advised to exercise the classical Yang's TCC for 40 minutes. Each session of Yang's TCC included a 10 minutes warm-up exercise (lower back and hamstring stretching, gentle calisthenics, and balance training), a 20-minutes TCC exercise, and a 10-minutes cool-down exercise. Each set of Yang's TCC consists of 64 successive postures. While practicing TCC, the TCC trainee kept the same pace in exercising the different postures of TCC in a sequence according to a pre-recorded tape to ensure that the same pace and sequence of postures were followed. After 3 months of TCC training, which consisted of 40 minutes' exercise each time, 7 times per week, a second hemodynamic measurements and blood sampling were performed using the same procedure. All procedures were performed in a bright and quiet room with a standardized temperature of 24 to $25^{\circ} \mathrm{C}$ and a standardized humidity of 54 to $55 \%$.

\section{Biochemistry and hormone analysis}

The biochemistry assays for the quantitative measurements of total cholesterol (TC) (Ektachem Clinical Chemistry Slides, Johnson \& Johnson), high-density lipoprotein-cholesterol (HDL-C) (INTEGRA 700, Roche), low-density lipoprotein-cholesterol (LDL-C) (INTEGRA 700 , Roche), triglyceride (TG) and fasting blood sugar (FBS) (Ektachem Clinical Chemistry Slides, Johnson \& Johnson) were performed on the blood sample obtained from each subject. The immunoradiometric assays for the quantitative measurement of insulin (DIAsource INSIRMA Kit, DIAsource ImmunoAssays S.A.), the radioimmunoassay for the quantitative measurement of aldosterone (DSL-8600 ACTIVE Aldosterone Coated-Tube Radioimmunoassay Kit, Diagnostic Systems Laboratories, Inc. USA) and the enzyme immunoassay for the quantitative determination of ET-1 (Kit Lot 998C Abl./Exp. 100908. CAT. NO. BI-20052. $12 \times 8$ TESTS, Biomedica Medizinprodukte $\mathrm{GmbH} \& \mathrm{Co}$ KG, A-1210 Wien, Divischgasse 4) in serum were also performed on the blood sample from each subject. After 3 months of TCC training, hemodynamic data, FBS and serum hormone levels of the TCC trainees were measured using the same methodology.

The homeostatic model assessment of insulin resistance (HOMAIR) was calculated by using the following formula: insulin level $(\mu \mathrm{U} / \mathrm{ml})$ $\mathrm{x}$ glycemia $(\mathrm{mmol} / \mathrm{l}) / 22.5[29,30]$. The QUICKI index was calculated as follows: $1 /[\log ($ insulin in $\mu \mathrm{U} / \mathrm{ml})+\log ($ glucose in $\mathrm{mg} / \mathrm{dl})]$ [31]. The triglyceride-to-HDL cholesterol ratio (TG/HDL-C) has been reported to be closely related to insulin resistance in adults $[32,33]$. These indices associated with insulin resistance were also examined in this study.

\section{Data analysis}

The t-test (SigmaPlot 12.5, SPSS Inc., Chicago, Illinois, USA) was employed to compare the general characteristics, hemodynamics, Lipid profile, FBS and circulating hormone levels between TCC trainees and the controls. The paired t-test was performed to compare the general characteristics, hemodynamics, Lipid profile, FBS and circulating hormone levels between before TCC training and after 3 months of TCC training in the TCC group. All data are presented as mean \pm SD A $p<0.05$ is considered statistically significant.

To correct for the baseline differences in the comparisons of lipid profile, FBS and circulating hormone levels, the percentage changes in these parameters in each subject before and after 3 months of TCC trainees were calculated by using the following formula:

$$
\% \mathrm{X}=\left[\left(\mathrm{X}_{\text {affer } 3 \text { monthTCC }}-\mathrm{X}_{\text {before }}\right) /\left(\mathrm{X}_{\text {before }}\right)\right] \times 100 \text {, }
$$

where $\mathrm{X}$ is the parameter to be compared. Correlation analysis was performed to analyze the relationship among the percentage changes in the parameters between before and after 3 months of TCC trainees.

\section{Results}

Twenty normal controls and 22 TCC trainees were included in this study. Table 1 displays the baseline characteristics of normal controls and TCC trainees before and after 3 months of TCC training. There was no significant difference in the baseline characteristics between these 2 groups of subjects except the age of TCC training.

Table 2 shows the hemodynamics, lipid profile, FBS and hormone levels before and after 3 months of TCC training. Between groups comparison indicated that there were no significant differences in the baseline data between the control group and the TCC group. However, after 3 months of TCC training, the pulse pressure (PP), HDL-C, QUICKI, ET-1, and aldosterone were significantly increased, whereas the serum insulin, HOMA, and $\log$ (HOMA) were significantly decreased in the TCC group, as compared with their counterparts in the control group.

Intra-group comparison showed that the PP, HOMA-IR and $\log$ (HOMA) were significantly decreased, while the TC, HDL-C, QUICKI, and aldosterone level were significantly increased 3 months after in the control group. Similarly, the PP, serum insulin, HOMA-IR, $\log$ (HOMA), and ET-1 were significantly decreased, while the HDL-C, QUICKI and aldosterone were significantly increased after 3 months of TCC training in the TCC group.

Table 3 shows that the \%FBS correlated significantly and negatively with the \%TG/HDL-C, \%QUICKI, and \%ET-1. In addition, the \%Insulin correlated significantly and positively with \%HOMA, and significantly and negatively with the \%QUICKI. Furthermore, the $\%$ HOMA correlated significantly and negatively with the \%QUICKI. 
Citation: Lu WA, Kuo CD (2015) The Changes in Serum Insulin, Aldosterone and Endothelin-1 Levels After 3 Months of Tai Chi Chuan Training in the Adults. J Diabetes Metab 6: 557. doi:10.4172/2155-6156.1000557

Page 3 of 5

\begin{tabular}{|c|c|c|c|c|}
\hline General characteristics & $\begin{array}{l}\text { Controls } \\
\text { Before TCC } \\
(\mathrm{N}=\mathbf{2 0})\end{array}$ & $\begin{array}{l}\text { Controls } \\
\text { After TCC } \\
(\mathrm{N}=\mathbf{2 0})\end{array}$ & $\begin{array}{c}\text { TCC trainees } \\
\text { Before TCC } \\
(\mathrm{N}=22)\end{array}$ & $\begin{array}{l}\text { TCC trainees } \\
\text { After TCC } \\
(\mathrm{N}=22)\end{array}$ \\
\hline Age (yrs) & $51.5 \pm 7.3$ & $5.5 \pm 7.3$ & $54.6 \pm 9.1$ & $54.6 \pm 9.1$ \\
\hline Gender (M/F) & 9/11 & 9/11 & $12 / 10$ & $12 / 10$ \\
\hline Body weight (kg) & $65.2 \pm 8.8$ & $65.2 \pm 8.0$ & $63.4 \pm 10.5$ & $62.9 \pm 10.3$ \\
\hline Body height $(\mathrm{cm})$ & $163.8 \pm 5.3$ & $163.8 \pm 5.3$ & $164.7 \pm 8.1$ & $164.7 \pm 8.1$ \\
\hline $\mathrm{BMI}\left(\mathrm{kg} / \mathrm{m}^{2}\right)$ & $24.2 \pm 2.9$ & $24.3 \pm 2.7$ & $23.2 \pm 2.7$ & $23.0 \pm 2.5$ \\
\hline Waist $(\mathrm{cm})$ & $83.8 \pm 9.3$ & $84.7 \pm 9.5$ & $82.1 \pm 9.9$ & $81.5 \pm 9.7$ \\
\hline Age of TCC (yrs) & 0 & 0 & 0 & $0.25 \pm 0.00^{\# t}$ \\
\hline
\end{tabular}

Data are presented as Mean \pm SD. BMI: body mass index; Values presented are Mean \pm SD. ${ }^{*} P<0.05$ vs. Controls (After TCC), ${ }^{\dagger} P<0.05$ vs. TCC trainees (Before TCC)

Table 1: Characteristics of Normal Controls and TCC Trainees Before and After 3 Months of TCC Training.

\begin{tabular}{|c|c|c|c|c|}
\hline $\begin{array}{l}\text { Hemodynamics and blood } \\
\text { parameters }\end{array}$ & $\begin{array}{c}\text { Controls } \\
\text { Before TCC } \\
(\mathrm{N}=20)\end{array}$ & $\begin{array}{c}\text { Controls } \\
\text { After TCC } \\
(\mathrm{N}=\mathbf{2 0})\end{array}$ & $\begin{array}{c}\text { TCC trainees } \\
\text { Before TCC } \\
(\mathrm{N}=22)\end{array}$ & $\begin{array}{c}\text { TCC trainees } \\
\text { After TCC } \\
(\mathrm{N}=\mathbf{2 2})\end{array}$ \\
\hline \multicolumn{5}{|l|}{ Hemodynamics } \\
\hline $\mathrm{SBP}(\mathrm{mmHg})$ & $117.3 \pm 15.5$ & $117.2 \pm 15.6$ & $123.7 \pm 21.0$ & $125.8 \pm 23.4$ \\
\hline $\mathrm{DBP}(\mathrm{mmHg})$ & $67.2 \pm 11.1$ & $72.4 \pm 10.8$ & $72.1 \pm 13.5$ & $76.6 \pm 11.4$ \\
\hline $\operatorname{MABP}(\mathrm{mmHg})$ & $83.9 \pm 11.4$ & $87.3 \pm 11.9$ & $89.3 \pm 15.1$ & $93.0 \pm 14.1$ \\
\hline $\mathrm{PP}(\mathrm{mmHg})$ & $50.1 \pm 12.2$ & $44.8 \pm 8.6^{*}$ & $51.6 \pm 13.6$ & $49.2 \pm 17.7^{\dagger}$ \\
\hline \multicolumn{5}{|l|}{ Lipid profile } \\
\hline TG (mg/dl) & $166.7 \pm 71.3$ & $167.4 \pm 72.6$ & $144.9 \pm 86.8$ & $137.7 \pm 90.2$ \\
\hline $\mathrm{TC}(\mathrm{mg} / \mathrm{dl})$ & $176.5 \pm 31.6$ & $179.0 \pm 32.6^{*}$ & $222.9 \pm 217.0$ & $184.6 \pm 23.4$ \\
\hline LDL-C (mg/dl) & $105.0 \pm 30.1$ & $105.8 \pm 29.6$ & $106.4 \pm 25.4$ & $111.1 \pm 24.0$ \\
\hline HDL-C (mg/dl) & $47.4 \pm 8.0$ & $48.6 \pm 8.6^{*}$ & $53.5 \pm 11.8$ & $54.6 \pm 10.7^{\dagger}$ \\
\hline HDL-C/TC & $0.28 \pm 0.06$ & $0.28 \pm 0.06$ & $0.29 \pm 0.09$ & $0.30 \pm 0.07$ \\
\hline TG/HDL-C & $3.65 \pm 1.73$ & $3.59 \pm 1.75$ & $3.02 \pm 2.22$ & $2.73 \pm 2.06$ \\
\hline \multicolumn{5}{|l|}{ FBS, Hormone and ET-1 } \\
\hline $\mathrm{FBS}(\mathrm{mmol} / \mathrm{l})$ & $5.1 \pm 1.5$ & $4.7 \pm 0.8$ & $6.5 \pm 3.4$ & $4.9 \pm 1.5$ \\
\hline Insulin $(\mu \mathrm{U} / \mathrm{ml})$ & $5.3 \pm 4.6$ & $3.7 \pm 3.5$ & $4.5 \pm 5.6$ & $2.7 \pm 6.8^{\dagger}$ \\
\hline HOMA-IR & $1.4 \pm 1.8$ & $0.8 \pm 0.9^{*}$ & $1.7 \pm 2.6$ & $0.5 \pm 1.1^{\# \dagger}$ \\
\hline $\log (\mathrm{MOMA}-\mathrm{IR})$ & $-0.08 \pm 0.43$ & $-0.29 \pm 0.45^{*}$ & $-0.24 \pm 0.70$ & $-0.61 \pm 0.48^{\#+}$ \\
\hline QUICKI & $0.41 \pm 0.07$ & $0.45 \pm 0.09^{*}$ & $0.46 \pm 0.14$ & $0.53 \pm 0.12^{\# \dagger}$ \\
\hline Aldosterone (pg/ml) & $78.4 \pm 37.7$ & $64.7 \pm 31.4^{*}$ & $62.0 \pm 23.8$ & $65.1 \pm 23.5^{\dagger}$ \\
\hline ET-1 (fmol/ml) & $0.63 \pm 0.45$ & $1.75 \pm 4.35$ & $2.14 \pm 4.51$ & $2.11 \pm 4.30^{\#+}$ \\
\hline
\end{tabular}

Data are presented as Mean \pm SD. SBP: systolic blood pressure; DBP: diastolic blood pressure; MABP: mean arterial blood pressure; PP: pulse pressure; TG: triglycerides; TC: total cholesterol; LDL-C: low-density lipoprotein-cholesterol; HDL-C:high-density lipoprotein-cholesterol; HDL-C/TC: ratio of HDL-C over TC; FBS: fasting blood sugar; ET-1: endothelin-1; HOMA-IR: fasting insulin $(\mu \mathrm{Ul} / \mathrm{ml}) \times$ fasting glucose $(\mathrm{mmol} / \mathrm{l}) / 22.5 ;$ QUICKI $=1 /[\log ($ insulin in $\mu \mathrm{U} / \mathrm{ml})+\mathrm{log}(\mathrm{glucose}$ in $\mathrm{mg} / \mathrm{dl})] .{ }^{*} P<0.05 \mathrm{vs}$. Controls (Before TCC); ${ }^{\#} P<0.01$ vs. Controls (After TCC); ${ }^{\dagger} P<0.05$ vs. TCC trainees (Before TCC).

Table 2: Hemodynamics, Lipid profile, BS and Hormone Levels of Normal Controls and TCC Trainees Before and After 3 Months of TCC Training.

\begin{tabular}{|c|c|c|c|c|c|c|c|c|}
\hline & $\%$ FBS & \%Insulin & \%HOMA & $\% \log (\mathrm{HOMA})$ & $\% \mathrm{TG} / \mathrm{HDL}-\mathrm{C}$ & \%QUICKI & \%Aldosterone & $\%$ ET-1 \\
\hline$\% H D L-C$ & 0.198 & 0.181 & 0.209 & 0.074 & -0.349 & -0.061 & -0.278 & -0.132 \\
\hline$\%$ FBS & & 0.135 & 0.205 & 0.067 & $-0.489^{*}$ & $-0.563^{*}$ & 0.206 & $-0.535^{*}$ \\
\hline \%Insulin & & & $0.992^{*}$ & 0.017 & -0.225 & $-0.575^{\star}$ & -0.203 & -0.037 \\
\hline \%HOMA & & & & 0.021 & -0.221 & $-0.618^{*}$ & -0.199 & -0.048 \\
\hline$\% \log (\mathrm{HOMA})$ & & & & & 0.156 & -0.341 & 0.207 & 0.071 \\
\hline$\% \mathrm{TG} / \mathrm{HDL}-\mathrm{C}$ & & & & & & 0.089 & -0.177 & 0.313 \\
\hline \%QUICKI & & & & & & & 0.002 & 0.309 \\
\hline \%Aldosterone & & & & & & & & -0.030 \\
\hline
\end{tabular}

Values are correlation coefficients. ${ }^{*} P<0.05$.

Table 3: Results of Correlation Analysis for The Percentage Changes in FBS, Indices of Insulin Resistance, and Related Hormone Levels After 3 Months of TCC Training in TCC Trainees.

\section{Discussion}

In the present study, we found that 3 months of TCC training can significantly increase the HDL-C, QUICKI, and aldosterone level, and significantly decrease serum insulin, HOMA, and $\log$ (HOMA), ET-1 in the TCC group, as compared with their counterparts before the beginning of TCC training. Moreover, there were some significant correlations among \%FBS, \%Insulin, \%Aldosterone, \%HOMA, \%QUICKI, and \%TG/HDL-C in the TCC group. These findings suggested that TCC might have an effect on the lipid and glucose metabolism, and the interplay among glucose-related hormones in its practitioners. 


\section{Effect of TCC on lipid profile}

The impact of physical exercise on HDL metabolism has been recognized as a major mechanism involved in the reduction of the risk of coronary artery disease. Some evidences have pointed that endurance exercise training can increase plasma HDL levels if the exercise training is sufficient [34-36]. Jafari et al. [37] demonstrated that exercise significantly increases plasma pre-b HDL and decrease plasma HDL-triglycerides. Couillard et al. [38] further showed that regular endurance exercise training may be particularly helpful in men with low HDL-cholesterol, since HDL-C concentration have been shown to increase with regular endurance exercise and, therefore, can contribute to a lower risk of coronary heart disease. Thus, not only endurance exercise, but also low-velocity and low-impact exercise such as TCC can increase the serum HDL-C in its practitioners. The mechanism underlying the increase in HDL-C after endurance exercise or 3 months of TCC is not clear yet.

\section{FBS, serum insulin, ET-1 and insulin resistance}

In this study we found that the serum aldosterone was significantly increased, while the serum insulin and ET-1 were significantly decreased by 3 months of TCC, as compared with their counterparts before TCC practice. The \%FBS had significant and negative correlation with either \%TG/HDL-C, \%QUICKI, or \%ET-1 in the TCC group, and the \%Insulin had significant and positive correlation with \%HOMA, but significant and negative correlation with \%QUICKI in the TCC trainees. However, the \%Aldosterone had no correlations with the percentages of changes in HDL-C, FBS, insulin, HOMA, etc., in this study. It seems that the ET-1 might have a role in the regulation of blood glucose, but the role of aldosterone is ambiguous. This is interesting and bewildering. In the literature, a direct relationship between plasma aldosterone levels, insulin resistance, and hyperinsulinemia has been reported in hypertensive patients [39]. In addition, aldosterone may be involved in the pathogenesis of vascular insulin resistance via oxidative stress because aldosterone can decreases insulin receptor substrate-1 expression via Src and reactive oxygen species stimulation by proteasome-dependent degradation in vascular smooth muscle cells [40]. Since aldosterone is regulated by sodium, potassium levels and plasma renin activity, it may not be meaningful to discuss the meaning of the change in the serum level of single aldosterone without simultaneous knowledge of sodium, potassium levels and plasma renin activity. Further studies are needed to elucidate the role of aldosterone in the regulation of serum insulin and insulin resistance.

It has been reported that insulin can modulates ET-1 levels in vivo [41], and ET-1 might impair insulin sensitivity [42] and inhibits plasma insulin responsiveness [13]. In this study, we found that both serum insulin and ET-1 level were decreased after 3 months of TCC in TCC trainees. Furthermore, we found that the \%FBS had significant and negative correlation with \%ET- 1 in the TCC trainees, and that there was no correlation between \%ET-1 and \%Insulin, \%HOMA, \%QUICKI or \%TG/HDL-C in this study. Thus, ET-1 might be involved in the regulation of blood sugar, but the exact role of ET-1 in the regulations of serum insulin and insulin resistance needs further studies to elucidate.

Aging is associated with the development of cardiovascular structural and functional alterations, which can explain the age-related increase in cardiovascular risk. Because endothelial dysfunction has been associated with advancing age [43], and circulating plasma ET-1 being a potent vasoconstrictor that might be mainly originate from vascular endothelial cells [44], it may be good to know that the increased production of ET-1 in vascular endothelial cells due to aging can be decreased by regular exercise. Maeda and associates [45] showed that regular aerobic-endurance exercise reduces plasma ET-1 concentration in older humans. Lewczuk et al. [46] demonstrated that plasma ET-1 concentration is decreased after cycling in healthy men. Therefore, it is comprehensible that the ET-1 level is decreased by 3 months of TCC training in this study. Since plasma level of ET-1 correlates with disease severity [47-49], the decrease in serum ET-1 level by 3 months of TCC may have beneficial effects on the cardiovascular systemand the metabolic function of the subjects.

Some people who went to the TCC center to learn TCC might have some cardiovascular or metabolic problems such as elevated blood pressures, elevated blood sugar, etc., and wanted to improve their health conditions through TCC practicing. That is the reason why the FBS of some TCC trainees was slightly elevated, though the FBS of the TCC trainees was not statistically different from that of the controls. That the basal health status of the TCC group might not be similar to that of the control group is one limitation of this study. Another limitation of this study is that the sample size of this study was not big enough. This might be the main reason why some of the results of this study are not easy to interpret.

\section{Conclusion}

TCC training for 3 months can increase the levels of ET-1 and aldosterone, and decrease serum insulin level and insulin resistance in the TCC trainees. The beneficial effects of TCC on the cardiovascular function and insulin sensitivity might become true through regulation of lipid and glucose metabolism, and the interplay among insulin, aldosterone, ET-1, and possibly others. The effects of 3 months of TCC on the lipid profile, FBS and related hormones deserve further investigations and validation.

\section{Acknowledgment}

This study was supported by the grant V98E2-014 of Taipei Veterans General Hospital, Taipei, TAIWAN

\section{References}

1. Lea and Febiger (1991) American College of Sports Medicine: Guidelines for Exercise Testing and Exercise Prescription. ( ${ }^{\text {th }}$ edn), Philadelphia, Williams and Wilkens.

2. Goble AJ, Hare DL, Macdonald PS, Oliver RG, Reid MA, et al. (1991) Effect of early programmes of high and low intensity exercise on physical performance after transmural acute myocardial infarction. Br Heart J 65: 126-131.

3. Lan C, Chen SY, Lai JS, Wong MK (1999) The effect of Tai Chi on cardiorespiratory function in patients with coronary artery bypass surgery. Med Sci Sports Exerc 31: 634-638.

4. Blumenthal JA, Rejeski WJ, Walsh-Riddle M, Emery CF, Miller H, et al. (1988) Comparison of high- and low-intensity exercise training early after acute myocardial infarction. Am J Cardiol 61: 26-30.

5. Bravo G, Gauthier P, Roy PM, Payette H, Gaulin P, et al. (1996) Impact of a 12-month exercise program on the physical and psychological health of osteopenic women. J Am Geriatr Soc 44: 756-762.

6. Xu D, Emoto N, Giaid A, Slaughter C, Kaw S, deWit D, et al. (1994) ECE-1: a membrane-bound metalloprotease that catalyzes the proteolytic activation of big endothelin-1. Cell 78: 473-485.

7. Yanagisawa M, Kurihara H, Kimura S, Tomobe $\mathrm{Y}$, Kobayashi M, et al. (1988) A novel potent vasoconstrictor peptide produced by vascular endothelial cells Nature 332: 411-415.

8. Vane JR, Anggård EE, Botting RM (1990) Regulatory functions of the vascular endothelium. N Engl J Med 323: 27-36.

9. Lerman A, Edwards BS, Hallett JW, Heublein DM, Sandberg SM, et al. (1991) Circulating and tissue endothelin immunoreactivity in advanced atherosclerosis. N Engl J Med 325: 997-1001. 
10. Takahashi K, Ghatei MA, Lam HC, O'Halloran DJ, Bloom SR (1990) Elevated plasma endothelin in patients with diabetes mellitus. Diabetologia 33: 306-310.

11. Idris I, Patiag D, Gray S, Donnelly R (2001) Tissue- and time-dependent effects of endothelin-1 on insulin-stimulated glucose uptake. Biochem Pharmacol 62: $1705-1708$.

12. Kao YS, Fong JC (2004) Thapsigargin and EGTA inhibit endothelin-1-induced glucose transport. J Biomed Sci 11: 206-213

13. Teuscher AU, Lerch M, Shaw S, Pacini G, Ferrari P, et al. (1998) Endothelin-1 infusion inhibits plasma insulin responsiveness in normal men. J Hypertens 16 : 1279-1284.

14. Kjaer A, Appel J, Hildebrandt P, Petersen CL (2004) Basal and exerciseinduced neuroendocrine activation in patients with heart failure and in normal subjects. Eur J Heart Fail 6: 29-39.

15. Kohno M, Yasunari K, Murakawa K, Yokokawa K, Horio T, et al. (1990) Plasma immunoreactive endothelin in essential hypertension. Am J Med 88: 614-618.

16. Shichiri M, Hirata Y, Ando K, Emori T, Ohta K, et al. (1990) Plasma endothelin levels in hypertension and chronic renal failure. Hypertension 15: 493-496.

17. Omland T, Lie RT, Aakvaag A, Aarsland T, Dickstein K (1994) Plasma endothelin determination as a prognostic indicator of 1 -year mortality after acute myocardial infarction. Circulation 89: 1573-1579.

18. Pekdemir H, Cicek D, Camsari A, Akkus MN, Cin VG, et al. (2004) The relationship between plasma endothelin-1, nitric oxide levels, and heart rate variability in patients with coronary slow flow. Ann Noninvasive Electrocardiol 9: 24-33.

19. Romagni P, Rossi F, Guerrini L, Quirini C, Santiemma V (2003) Aldosterone induces contraction of the resistance arteries in man. Atherosclerosis 166: 345349.

20. Tostes RC, Touyz RM, He G, Chen X, Schiffrin EL (2002) Contribution of endothelin-1 to renal activator protein-1 activation and macrophage infiltration in aldosterone-induced hypertension. Clin Sci (Lond) 103 Suppl 48: 25S-30S.

21. Lan C, Lai JS, Wong MK, Yu ML (1996) Cardiorespiratory function, flexibility, and body composition among geriatric Tai Chi Chuan practitioners. Arch Phys Med Rehabil 77: 612-616

22. Lai JS, Lan C, Wong MK, Teng SH (1995) Two-year trends in cardiorespiratory function among older Tai Chi Chuan practitioners and sedentary subjects. J Am Geriatr Soc 43: 1222-1227.

23. Lan C, Lai JS, Chen SY, Wong MK (1998) 12-month Tai Chi training in the elderly: its effect on health fitness. Med Sci Sports Exerc 30: 345-351.

24. Wolfson L, Whipple R, Derby C, Judge J, King M, et al. (1996) Balance and strength training in older adults: intervention gains and Tai Chi maintenance. $J$ Am Geriatr Soc 44: 498-506.

25. Jin $P$ (1992) Efficacy of Tai Chi, brisk walking, meditation, and reading in reducing mental and emotional stress. J Psychosom Res 36: 361-370.

26. Sun J, Buys N, Jayasinghe R (2014) Effects of community-based meditative Tai Chi programme on improving quality of life, physical and mental health in chronic heart-failure participants. Aging Ment Health 18: 289-295.

27. Xiong KY, He H, Ni GX (2013) Effect of skill level on cardiorespiratory and metabolic responses during Tai Chi training. Eur J Sport Sci 13: 386-391.

28. China Publications Center (1983) CHINA SPORTS: Simplified "Taijiquan". (2nd edn), Beijing, pp. 1-5

29. Puchau B, Hermsdorff HH, Zulet MA, Martínez JA (2009) DDAH2 mRNA expression is inversely associated with some cardiovascular risk-related features in healthy young adults. Dis Markers 27: 37-44.

30. Salgado AL, Carvalho Ld, Oliveira AC, Santos VN, Vieira JG, et al. (2010) Insulin resistance index (HOMA-IR) in the differentiation of patients with nonalcoholic fatty liver disease and healthy individuals. Arq Gastroenterol 47: 165169.
31. Katz A, Nambi SS, Mather K, Baron AD, Follmann DA, et al. (2000) Quantitative insulin sensitivity check index: a simple, accurate method for assessing insulin sensitivity in humans. J Clin Endocrinol Metab 85: 2402-2410.

32. McLaughlin T, Abbasi F, Cheal K, Chu J, Lamendola C, et al. (2003) Use of metabolic markers to identify overweight individuals who are insulin resistant. Ann Intern Med 139: 802-809.

33. Li C, Ford ES, Meng YX, Mokdad AH, Reaven GM (2008) Does the association of the triglyceride to high-density lipoprotein cholesterol ratio with fasting serum insulin differ by race/ethnicity? Cardiovasc Diabetol 7: 4

34. Després JP, Pouliot MC, Moorjani S, Nadeau A, Tremblay A, et al. (1991) Loss of abdominal fat and metabolic response to exercise training in obese women. Am J Physiol 261: E159-167.

35. Durstine JL, Haskell WL (1994) Effects of exercise training on plasma lipids and lipoproteins. Exerc Sport Sci Rev 22: 477-521.

36. Hardman AE (1999) Physical activity, obesity and blood lipids. Int J Obes Relat Metab Disord 23 Suppl 3: S64-71.

37. Jafari M, Leaf DA, Macrae H, Kasem J, O'conner P, et al. (2003) The effects of physical exercise on plasma prebeta-1 high-density lipoprotein. Metabolism 52: 437-442.

38. Couillard C, Després JP, Lamarche B, Bergeron J, Gagnon J, et al. (2001) Effects of endurance exercise training on plasma HDL cholesterol levels depend on levels of triglycerides: evidence from men of the Health, Risk Factors, Exercise Training and Genetics (HERITAGE) Family Study. Arterioscler Thromb Vasc Biol 21: 1226-1232.

39. Colussi G, Catena C, Lapenna R, Nadalini E, Chiuch A, et al. (2007) Insulin resistance and hyperinsulinemia are related to plasma aldosterone levels in hypertensive patients. Diabetes Care 30: 2349-2354

40. Hitomi H, Kiyomoto H, Nishiyama A, Hara T, Moriwaki K, et al. (2007) Aldosterone suppresses insulin signaling via the downregulation of insulin receptor substrate-1 in vascular smooth muscle cells. Hypertension 50: 750755 .

41. Wolpert HA, Steen SN, Istfan NW, Simonson DC (1993) Insulin modulates circulating endothelin-1 levels in humans. Metabolism 42: 1027-1030.

42. Ferri C, Carlomagno A, Coassin S, Baldoncini R, Cassone Faldetta MR et al. (1995) Circulating endothelin-1 levels increase during euglycemic hyperinsulinemic clamp in lean NIDDM men. Diab Care 18: 226-233.

43. Taddei S, Virdis A, Mattei P, Ghiadoni L, Gennari A, et al. (1995) Aging and endothelial function in normotensive subjects and patients with essential hypertension. Circulation 91: 1981-1987.

44. Dietmann A, Lackner P, Helbok R, Spora K, Issifou S, et al. (2008) Opposed circulating plasma levels of endothelin- 1 and C-type natriuretic peptide in children with Plasmodium falciparum malaria. Malar J 7: 253.

45. Maeda S, Tanabe T, Miyauchi T, Otsuki T, Sugawara J, et al. (2003) Aerobic exercise training reduces plasma endothelin-1 concentration in older women. Appl Physiol (1985) 95: 336-341.

46. Lewczuk P, Söhnchen N, Kele H, Reimers CD, Ehrenreich H (2003) Endothelin-1 concentration in plasma is increased after jogging but decreased after cycling in healthy men. Clin Exp Med 2: 166-170.

47. Cacoub P, Dorent R, Maistre G, Nataf P, Carayon A, et al. (1993) Endothelin-1 in primary pulmonary hypertension and the Eisenmenger syndrome. Am J Cardiol 71: 448-450

48. Ishikawa S, Miyauchi T, Ueno H, Ushinohama H, Sagawa K, et al. (1995) Influence of pulmonary blood pressure and flow on endothelin-1 production in humans. J Cardiovasc Pharmacol 26 Suppl 3: S429-433.

49. Yoshibayashi M, Nishioka K, Nakao K, Saito Y, Matsumura M, et al. (1991) Plasma endothelin concentrations in patients with pulmonary hypertension associated with congenital heart defects. Evidence for increased production of endothelin in pulmonary circulation. Circulation 84: 2280-2285. 